\title{
Perfil dos pacientes diagnosticados com displasia do desenvolvimento do quadril ${ }^{*}$
}

\section{Profile of Patients Diagnosed with Developmental Dysplasia of the Hip}

\author{
Renan de Oliveira Barbosa ${ }^{1}$ Elaine Pinto Albernaz ${ }^{1}$ \\ ${ }^{1}$ Faculdade de Medicina, Universidade Católica de Pelotas, Pelotas, \\ RS, Brasil \\ Endereço para correspondência Renan de Oliveira Barbosa, Master, \\ Faculdade de Medicina, Universidade Católica de Pelotas, Pelotas, RS, \\ Brasil (e-mail: renanoliveirabarbosa@bol.com.br).
}

Rev Bras Ortop 2019;54:497-502.

\section{Resumo \\ Palavras-chave \\ - luxação congênita de quadril \\ - displasia do desenvolvimento do quadril \\ - triagem neonatal}

Abstract
Objetivo Descrever o perfil dos pacientes com displasia do desenvolvimento do quadril (DDQ), diagnosticados por meio de exame físico e ultrassonográfico, com a implantação do protocolo de atenção e rastreio de DDQ.

Métodos Estudo transversal que incluiu os portadores de DDQ nascidos de janeiro de 2014 a dezembro de 2016, na cidade de Pelotas, Sul do Brasil, que considerou os fatores etnia, sexo, peso ao nascer, posição fetal, lado de ocorrência, idade gestacional, idade materna e histórico familiar. Os dados de prontuário foram comparados com as características da população geral por meio do Sistema de Informação sobre Nascidos Vivos (Sinasc).

Resultados Foram identificados 33 portadores de DDQ, a maioria do sexo feminino, que mostrou uma probabilidade quatro vezes maior de apresentar a patologia $(p<0,001)$, e o lado mais acometido foi o esquerdo. Os recém-nascidos com apresentação pélvica tiveram uma probabilidade 15 vezes maior de ter DDQ $(p<0,001)$. Não foi encontrada associação estatisticamente significativa com os seguintes fatores avaliados: peso ao nascer, idade gestacional, etnia e idade materna. Um total de 21 recém-nascidos necessitaram de tratamento imediato do quadril; a ecografia demonstrou classificação Ilb ou maior, pelo método de Graf, ou a radiografia mostrou luxação nos portadores de DDQ com mais de seis meses de idade.

Conclusão O rastreio de DDQ é essencial em todos os recém-nascidos, e o exame físico, quando alterado, deve ser complementado com o ultrassonográfico para evitar o diagnóstico tardio da doença.

Objective To describe the profile of patients with developmental dysplasia of the hip $(\mathrm{DDH})$, diagnosed by means of physical and ultrasound examination, with the implementation of a protocol for the treatment and follow-up of DDH.

Methods A cross-sectional study with DDH patients born between January 2014 and December 2016, in the city of Pelotas, Southern Brazil. Ethnicity, sex, birth weight,
Trabalho desenvolvido no Mestrado em Saúde da criança e adolescente da Universidade Católica de Pelotas, Pelotas, RS Brasil. Publicado Originalmente por Elsevier Editora Ltda. recebido

20 de Dezembro de 2017 aceito

21 de Fevereiro de 2018
DOI https://doi.org/

10.1016/j.rbo.2018.02.005. ISSN 0102-3616.
Copyright $\odot 2019$ by Sociedade Brasileira License terms de Ortopedia e Traumatologia. Published by Thieme Revinter Publicações Ltda, Rio de Janeiro, Brazil 


\section{Keywords}

- congenital hip dislocation

- developmental dysplasia of the hip

- neonatal screening fetal presentation, affected side of the hip, gestational age, maternal age, and family history were considered. The data on the medical records were compared with the characteristics of the general population through the Brazilian National Information System on Live Births (Sistema de Informação sobre Nascidos Vivos [SINASC]).

Results A total of $33 \mathrm{DDH}$ patients were identified, mostly female, with a four-fold higher probability of having the condition $(p<0.001)$; the left was the most affected side. No statistically significant association was found regarding the following factors: birth weight, gestational age, ethnicity, and maternal age. The newborns in breech presentation had a 15-fold higher probability of having $\mathrm{DDH}(p<0.001)$. A total of 21 newborns required immediate treatment of the hips, since the ultrasound showed a Graf classification of Ilb or higher, by the Graf method, or the radiography showed dislocation in DDH patients older than 6 months of age.

Conclusion Screening for DDH is essential in all newborns; physical examinations revealing alterations must be complemented with ultrasound imaging to avoid the delayed diagnosis of the condition.

\section{Introdução}

Displasia do desenvolvimento do quadril (DDQ) é o termo que descreve todas as alterações no quadril do recém-nascido (RN), e engloba desde os quadris instáveis até os luxados. Substitui a classificação anterior "luxação congênita do quadril”, a qual, como o próprio nome diz, engloba somente os quadris luxados. ${ }^{1}$

O rastreio de DDQ, no RN, atualmente é feito pela manobra descrita por Ortolani, ${ }^{24}$ que avalia se há presença de luxação e instabilidade do quadril e, em alguns casos, pelo exame ultrassonográfico, por meio do método descrito por Graf, que classifica o quadril em maduro e estável (tipo I), imaturo (tipo Ila), imaturo-instável (tipo IIb), instável (tipo IIc), e luxado (tipos III e IV). ${ }^{2,3}$ Quando o exame físico ou ecográfico é positivo, usa-se um suspensório descrito por Pavlik, órtese flexível que mantém o quadril em uma posição de flexão de $90^{\circ}$ a $110^{\circ}$, e abdução na zona de segurança, entre $30^{\circ}$ e $60^{\circ} .4$ O tratamento apresenta uma taxa de sucesso de $86 \%$ a 99\%. ${ }^{1,5,6}$ Esse índice de sucesso é facilmente explicado por estarem preservadas as estruturas anatômicas do quadril no $\mathrm{RN}$, e a redução precoce ocasiona o crescimento normal da articulação.

Os casos diagnosticados e tratados tardiamente ainda são a principal causa de artrite precoce do quadril, e resultam em dor, incapacidade funcional e artroplastia total do quadril em adultos jovens. ${ }^{1}$ Metade dos casos de DDQ diagnosticados e tratados tardiamente apresentará algum grau de degeneração da articulação coxofemoral entre os 16 e 31 anos. $^{7}$

As características físicas do RN e da mãe podem acarretar uma maior probabilidade de DDQ, e aumentam em cerca de $60 \%$ a sua prevalência. Os fatores descritos relacionados a esse aumento são: sexo feminino, etnia branca, peso ao nascer acima de $4.000 \mathrm{~g}$, presença de histórico familiar positivo, idade gestacional acima de 40 semanas, mãe primigesta, idade materna acima de 35 anos, alterações morfológicas dos pés, e posição fetal pélvica. Ocorre um aumento ainda maior quando há associação desses fatores. ${ }^{8-10}$

A proposta deste estudo foi traçar o perfil dos pacientes portadores de DDQ na cidade de Pelotas e implantar um protocolo para rastreio da patologia.

\section{Material e Métodos}

Trata-se de um estudo transversal, analítico, feito no único serviço de referência em tratamento das enfermidades ortopédicas pediátricas da cidade de Pelotas, RS. Foram avaliados os prontuários dos pacientes portadores de DDQ atendidos de janeiro de 2014 a dezembro de 2016, no Ambulatório de Ortopedia Infantil da nossa instituição, e, a partir de um questionário elaborado pelos autores, avaliaram-se as variáveis que são descritas como associadas ao aumento de prevalência de DDQ: etnia, sexo, idade materna, paridade, tempo de gestação, peso ao nascer, histórico familiar, posição fetal e malformações ortopédicas associadas.

As características dos RNs que compuseram a amostra foram comparadas àquelas dos nascidos vivos em Pelotas no período do estudo. Os dados desses foram obtidos no Sistema de Informação sobre Nascidos Vivos (Sinasc), visto que essas informações são rotineiramente enviadas pelas maternidades à Secretaria de Saúde do município. Os portadores de malformações do tubo neural, artrogripose, síndromes e doenças neuromusculares foram excluídos. O estudo foi previamente aprovado pelo Comitê de Ética em Pesquisa da instituição sob o número CAAE: 62063116.3.0000.5339.

Foi feita análise descritiva dos nascimentos ocorridos na cidade de Pelotas de janeiro de 2014 a dezembro de 2016, conforme as características que constam no Sinasc, assim como descrição dos casos de DDQ atendidos no serviço de referência. Para as variáveis que constavam tanto no Sinasc quanto nos prontuários, foi feita análise bivariada, e usou- 
se o teste do qui-quadrado ou o teste de Fisher, quando havia indicação. Para as análises, foi usado o Programa Epi Info 7.2. Foi considerado o nível de significância estatística de $95 \%$.

\section{Resultados}

As características dos nascimentos constantes no Sinasc podem ser observadas na tabela 1. De 2014 a 2016, nasceram 14.106 crianças na cidade de Pelotas, uma média de 4.702 nascimentos ao ano.

Foram detectados 35 portadores de DDQ atendidos no serviço de referência. Dois foram excluídos da amostra por não terem nascido em Pelotas. Dos 33 remanescentes, 21 necessitaram de tratamento imediato dos quadris, e a ecografia demonstrou classificação IIb ou maior, pelo método de Graf, ou a radiografia mostrou luxação nos portadores de DDQ com mais de seis meses de idade. Nos outros 12, que,

Tabela 1 Perfil dos recém-nascidos da cidade de Pelotas, RS Sistema de Informacões Sobre Nascidos Vivos (Sinasc, 2014 a 2016)

\begin{tabular}{|l|l|}
\hline Características & $\mathbf{n}(\%)$ \\
\hline Sexo & \\
\hline Masculino & $7.185(50,93)$ \\
\hline Feminino & $6.920(49,06)$ \\
\hline Etnia ${ }^{\text {a }}$ & \\
\hline Branca & $6.207(79,63)$ \\
\hline Parda & $489(6,27)$ \\
\hline Negra & $1.090(13,98)$ \\
\hline Outras & $04(0,05)$ \\
\hline Peso & \\
\hline Abaixo de 3.000 g & $4.802(34,18)$ \\
\hline $3.000-3.999 \mathrm{~g}$ & $8.564(60,95)$ \\
\hline Acima de 4.000 g & $683(4,86)$ \\
\hline Duração da gestação & \\
\hline Até 37 semanas & $2.064(15,60)$ \\
\hline De 37 a 41 semanas & $1.0887(82,29)$ \\
\hline Acima de 41 semanas & $279(2,10)$ \\
\hline Idade da mãe & \\
\hline Até 19 anos & $2.042(14,47)$ \\
\hline $19-34$ anos & $9.966(70,65)$ \\
\hline 35 anos e acima & $2.097(14,86)$ \\
\hline Anomalias congênitas & \\
\hline Sim & $98(0,72)$ \\
\hline Não & $12.492(92,80)$ \\
\hline Não informado & $871(6,47)$ \\
\hline TOTAL & $14.106(100)$ \\
\hline
\end{tabular}

Nota: ${ }^{\mathrm{E} E m}$ 2016, não foram coletados dados relativos à etnia; para as demais variáveis, o percentual máximo de dado ignorado foi de $6,2 \%$. pelo exame ultrassonográfico, eram do tipo Ila, foi feito o acompanhamento, com reconsulta em quatro semanas, inclusive novo exame físico e de imagem. As características dos RNs com DDQ encontram-se na tabela 2.

A análise bivariada entre características maternas e do RN e a DDQ mostrou associação estatisticamente significativa em relação ao sexo da criança. As meninas mostraram uma probabilidade quase 4 vezes maior de apresentar DDQ (razão de prevalência $[R P]=3,86$; intervalo de confiança de $95 \%$ [IC95\%]: 1,68-8,88; $p<0,001)$. As variáveis peso ao nascer $(p=0,90)$, idade gestacional $(p=0,16)$, etnia $(p=0,53)$ e idade materna $(p=0,59)$ não mostraram associação estatisticamente significativa com o desfecho. A etnia só foi avaliada para os nascidos em 2014 e 2015, pois esse dado não constava no Sinasc para o ano de 2016. Os RNs de apresentação pélvica tiveram uma probabilidade 15 vezes maior de desenvolver $\operatorname{DDQ}(\mathrm{RP}=15,30$; IC95\%: 7,57-30,92; $p<0,001)$.

Quatro crianças apresentavam anomalias ortopédicas associadas: pé torto congênito e pé torto postural; duas nasceram com retrocurvato do joelho.

\section{Discussão}

Uma das limitações deste estudo foi o pequeno número de casos identificados, o que pode dificultar a avaliação de algumas associações. Além disso, algumas crianças podem não ter sido levadas ao serviço de referência por não terem sido devidamente examinadas ao nascimento. Apesar desse viés de seleção em potencial, a ocorrência encontrada de 2,3 casos por 1.000 nascimentos é semelhante à descrita na literatura. Há uma enorme variação da incidência de DDQ; ela depende da etnia, dos hábitos e da região geográfica da população. A articulação coxofemoral do RN apresenta uma incidência de luxação de 2/1.000 e instabilidade de 10/1.000 nascimentos. ${ }^{1}$ Há diferença entre as populações: é pouco frequente na etnia negra, e altamente prevalente nos índios americanos (76/1.000) e esquimós (25-40/1.000). ${ }^{11}$

Wynne-Davies $^{12}$ inferiu que a causa da DDQ era uma união da instabilidade devido a um aumento de laxidão da articulação coxofemoral com fatores ambientais que propiciam o aparecimento da patologia. Uma vez que os meninos são menos afetados, acredita-se haver uma causa hormonal ou uma laxidão articular menos acentuada no sexo masculino. ${ }^{12,13} \mathrm{O}$ presente estudo encontrou uma prevalência maior em meninas, achado semelhante ao de estudos anteriores. 2,9,11,13,14

É sabido que entre 60\% e 93\% dos portadores de DDQ apresentam fatores associados à patologia. ${ }^{15-17} \mathrm{~A}$ posição pélvica aumentaria o risco em 5-16 vezes. ${ }^{8,9,17}$ Neste estudo, a posição pélvica foi mencionada em $36 \%$ dos RNs com DDQ e aumentou significativamente a probabilidade de DDQ (15 vezes mais risco). Quanto ao histórico familiar, a literatura indica que ele aumenta a incidência de DDQ de 12 para 68 por 1.000 nascimentos; ${ }^{3,6,18-20} 24 \%$ da amostra do presente estudo tinha histórico familiar de DDQ. A etnia negra é mencionada como fator protetor, e é descrita uma incidência três vezes menor do que na branca, ${ }^{10}$ mas, na amostra avaliada, essa associação não se confirmou. A associação com peso ao nascimento também não foi estatisticamente significativa, 
Tabela 2 Perfil dos recém-nascidos portadores de displasia do desenvolvimento do quadril $(n=33)$

\begin{tabular}{|c|c|}
\hline \multicolumn{2}{|l|}{ Características \% } \\
\hline Sexo & \\
\hline Masculino & 21,21 \\
\hline Feminino & 78,78 \\
\hline \multicolumn{2}{|l|}{ Etnia } \\
\hline Branca & 69,69 \\
\hline Parda & 12,12 \\
\hline Negra & 9,09 \\
\hline Outras & 9,09 \\
\hline \multicolumn{2}{|l|}{ Idade materna } \\
\hline Até 19 anos & 9,09 \\
\hline Entre 20 e 34 anos & 69,69 \\
\hline Maior do que 35 anos & 15,15 \\
\hline Ignorada & 6,06 \\
\hline \multicolumn{2}{|l|}{ Primeiro filho } \\
\hline Sim & 63,63 \\
\hline Não & 27,27 \\
\hline Não informado & 9,09 \\
\hline \multicolumn{2}{|l|}{ Idade gestacional } \\
\hline Até 37 semanas & 18,18 \\
\hline De 37 a 41 semanas & 66,66 \\
\hline Acima de 41 semanas & 6,06 \\
\hline Ignorada & 9,09 \\
\hline \multicolumn{2}{|l|}{ Posição fetal } \\
\hline Cefálica & 57,57 \\
\hline Pélvica & 36,36 \\
\hline Ignorada & 6,06 \\
\hline \multicolumn{2}{|l|}{ Histórico familiar } \\
\hline Presente & 24,24 \\
\hline Ausente & 57,57 \\
\hline Ignorado & 18,18 \\
\hline \multicolumn{2}{|l|}{ Lado acometido } \\
\hline Direito & 12,12 \\
\hline Esquerdo & 57,57 \\
\hline Bilateral & 21,21 \\
\hline Ignorado & 9,09 \\
\hline \multicolumn{2}{|l|}{ Peso ao nascer } \\
\hline Abaixo de $3.000 \mathrm{~g}$ & 30,30 \\
\hline 3.000-3.999g & 54,54 \\
\hline Acima de $4.000 \mathrm{~g}$ & 6,06 \\
\hline Ignorado & 9,09 \\
\hline \multicolumn{2}{|c|}{ Anomalia ortopédica associada } \\
\hline Sim & 72,72 \\
\hline Não & 12,12 \\
\hline Não informado & 15,15 \\
\hline TOTAL & 100,00 \\
\hline
\end{tabular}

embora a literatura indique o peso ao nascimento superior a $4.000 \mathrm{~g}$ como fator de risco, e o inferior a $2.500 \mathrm{~g}$ como fator de proteção. ${ }^{8,9}$ A informação sobre paridade não constava nos dados do Sinasc, mas foi citada uma chance de duas a quatro vezes maior de o RN de primeira gestação apresentar DDQ em relação aos RNs de mães que já têm dois ou mais filhos. 8,17,20 Isso ocorre pelo fato de o útero ter um tamanho menor na primeira gestação. ${ }^{6,14,15,18,21}$ No presente estudo, a maioria dos casos era de filhos de primigestas (64\%).

Outros fatores descritos são a presença de oligodrâmnio (quatro vezes mais chance de ocorrência de DDQ); no presente estudo, um caso apresentou oligodrâmnio ${ }^{6,8}$ e pós-datismo. ${ }^{8,22}$ De acordo com as referências, a idade materna apresenta associação direta com a ocorrência de DDQ e é duas vezes mais frequente quando essa for superior a 35 anos. $^{8} \mathrm{Na}$ amostra avaliada, a idade não foi um fator que influenciou a presença de DDQ.

Foram identificadas malformações associadas em $12 \%$ da amostra, fator concordante com outros autores que descrevem essa associação. ${ }^{8,9,21,23}$ o lado esquerdo foi o mais acometido (58\%), e isso está de acordo com a literatura. 9,15,18

0 rastreio da patologia, a qual pode causar dor, limitação funcional e laboral, quando não tratada, é feito há bastante tempo. Em 1937, o pediatra italiano Marino Ortolani ${ }^{24}$ descreveu a manobra homônima, que consistia em fazer flexão e abdução do quadril. Quando ele se encontrava luxado, sentiase um ressalto, dado que o movimento descrito reduz a luxação. ${ }^{21,24}$ Von Rosen, ${ }^{25}$ em 1957, introduziu, na Suécia, o rastreio de rotina em todos os RNs com a manobra descrita por Ortolani, fato que reduziu drasticamente os diagnósticos tardios da doença. ${ }^{21,25}$ Atualmente, a manobra é unanimidade em todo o mundo, e é indicada para todos os RNs. A discussão é sobre quando deve ser feito um exame de imagem complementar (ultrassonografia) para minimizar o não diagnóstico de DDQ e os diagnósticos tardios da doença. ${ }^{26}$

Sabe-se que, com o diagnóstico precoce, antes dos seis meses de idade, e o subsequente uso do suspensório de Pavlik, o tratamento apresenta um índice de sucesso de $90 \% .^{6}$ Isso porque a articulação coxofemoral do RN, apesar de luxada, não se apresenta morfologicamente alterada, e a órtese faz com que a articulação reduza, se estabilize e, a partir do tratamento, apresente um desenvolvimento normal, na maioria dos casos.

O índice de complicações com o uso do suspensório é baixo, menor do que $1 \%$. A maior complicação do tratamento com suspensório é a necrose avascular da epífise femoral. Pode ocorrer também paralisia do nervo femoral, com remissão total da paralisia com a retirada do suspensório. ${ }^{14}$ Já o não diagnóstico ou o diagnóstico tardio, pós-idade da marcha (12 meses), faz com que haja uma degeneração precoce da articulação, acarretando um índice de $86 \%$ de indicação cirúrgica nos casos não diagnosticados até os 10 meses. ${ }^{7,22}$ Além disso, sabe-se que entre $25 \%$ e $40 \%$ dos casos precoces de osteoartrite do quadril são secundários à DDQ tratada de forma tardia ou negligenciada. ${ }^{27}$ Dos casos tardios que necessitam de cirurgia (osteotomia pélvica ou femoral), 44\% apresentarão algum grau de artrose entre os 16 e os 31 anos de idade. ${ }^{7}$ Aos 33 anos, póstratamento da DDQ tardia, 50\% dos casos irão apresentar artrite 
moderada ou grave, e $14 \%$ provavelmente já terão feito artroplastia total do quadril (ATQ); aos 45 anos, pós-cirurgia, 54\% já irão ter feito cirurgia de ATQ e 1/3 dos casos remanescentes irá apresentar coxartrose. $^{15}$

O método preferido de rastreio para o diagnóstico de DDQ permanece controverso. Atualmente, os métodos de rastreio incluem o exame físico e a ultrassonografia (US). 0 exame radiográfico só é indicado em crianças com mais de quatro meses. $^{18}$

Mahan et $\mathrm{al}^{6}$ descreveram três modelos de rastreamento de DDQ: o exame físico universal (manobras de Ortolani e Barlow); a US nos casos com exame físico positivo, posição pélvica e histórico familiar; e o modelo da US universal, que seria a US em todos os RNs. O estudo desses autores demonstrou que a chance de ter uma degeneração precoce do quadril com consequente artrite era maior nos casos não submetidos a US em comparação com os outros dois modelos, submetidos a US. Na comparação da US universal com a US com exame físico positivo, ou fatores de risco, esses autores perceberam aumento de falso-positivo no modelo universal. Já a presença de casos tardios ou degeneração precoce da articulação não mudou nos dois grupos. ${ }^{6}$ Schams et al $^{3}$ recomendam o modelo universal, pois a combinação de dois fatores de risco não mostrou evidência de maior risco de DDQ. Bache et $\mathrm{al}^{14}$ recomendam a US em todos os RNs do sexo feminino, e, para o sexo masculino, somente naqueles com fator de risco para DDQ. Woodacre et al ${ }^{17}$ observaram que somente histórico familiar e parto ou posição pélvica eram os fatores de risco associados à DDQ no sexo masculino.

Conforme recomendações da Pediatric Orthopaedic Society of North America (POSNA), o exame físico é fundamental, é feito primeiramente pelo pediatra, e, se positivo, o paciente é indicado ao ortopedista para esclarecer ou confirmar o diagnóstico de DDQ. Assim, o exame físico é superior ao de imagem (US). ${ }^{26,28}$ A US deve ser usada nos quadris com alto risco para DDQ ou com exame físico positivo. A concordância do exame físico com a ecografia é de $87,5 \%{ }^{27}$ Sabe-se que entre $60 \%$ e $80 \%$ das anormalidades do quadril dos RNs detectadas no exame físico resolvem-se em 2 a 8 semanas, assim como $90 \%$ das alterações encontradas em USs do quadril em RNs. ${ }^{14,26}$

No Brasil, o Ministério da Saúde (MS) recomenda a manobra de Ortolani nos dois primeiros dias de vida e nas consultas de puericultura subsequentes, e a US é recomendada quando o resultado da manobra de Ortolani for positivo, na presença de histórico familiar, apresentação pélvica, torcicolo congênito, ou se houver malformações dos pés. ${ }^{29}$

\section{Conclusão}

Apesar de o método de rastreio considerado ideal pelos autores do presente estudo ser a US do quadril em todos os RNs do sexo feminino e nos meninos que apresentarem uma das seguintes três características: resultado positivo da manobra de Ortolani, apresentação pélvica e histórico familiar, na cidade de Pelotas, assim como na maior parte do Brasil, esse modelo é inviável devido ao custo, à logística e à dificuldade de conseguir profissionais e aparelhagem de US para atender à demanda que esse rastreio geraria. Recomenda-se seguir efetivamente o protocolo do MS para evitar casos tardios de DDQ no nosso meio. Todas as maternidades devem seguir as suas diretrizes: manobra de Ortolani nos dois primeiros dias de vida e nas consultas de puericultura subsequentes, e US quando a manobra for positiva, na presença de histórico familiar, apresentação pélvica, torcicolo congênito ou malformações dos pés.

\section{Conflitos de Interesse}

Os autores declaram não haver conflitos de interesse.

\section{Referências}

1 Guarniero R. Displasia do desenvolvimento do quadril: atualização. Rev Bras Ortop 2010;45(02):1-9

2 Omeroğlu $\mathrm{H}$. Use of ultrasonography in developmental dysplasia of the hip. JChild Orthop 2014;8(02):105-113

3 Schams M, Labruyère R, Zuse A, Walensi M. Diagnosing developmental dysplasia of the hip using the Graf ultrasound method: risk and protective factor analysis in 11,820 universally screened newborns. Eur J Pediatr 2017;176(09):1193-1200

4 Ishida A, Kuwajima SS. Propedêutica ortopédica do quadril pediátrico. In: Leite NM, Faloppa F, eds. Propedêutica ortopédica e traumatológica. Porto Alegre: Artes Médicas; 2013:381-5

5 Woodacre T, Dhadwal A, Ball T, Edwards C, Cox PJ. The costs of late detection of developmental dysplasia of the hip. JChild Orthop 2014;8(04):325-332

6 Mahan ST, Katz JN, Kim YJ. To screen or not to screen? A decision analysis of the utility of screening for developmental dysplasia of the hip. J Bone Joint Surg Am 2009;91(07):1705-1719

7 Moraleda L, Albiñana J, Salcedo M, Gonzalez-Moran G. [Dysplasia in the development of the hip]. Rev Esp Cir Ortop Traumatol 2013; 57(01):67-77

8 Chan A, McCaul KA, Cundy PJ, Haan EA, Byron-Scott R. Perinatal risk factors for developmental dysplasia of the hip. Arch Dis Child Fetal Neonatal Ed 1997;76(02):F94-F100

9 Mulpuri K, Schaeffer EK, Andrade J, et al; IHDI Study Group. What risk factors and characteristics are associated with late-presenting dislocations of the hip in infants? Clin Orthop Relat Res 2016; 474(05):1131-1137

10 Loder RT, Shafer C. The demographics of developmental hip dysplasia in the Midwestern United States (Indiana). JChild Orthop 2015;9(01):93-98

11 Thaler M, Biedermann R, Lair J, Krismer M, Landauer F. Costeffectiveness of universal ultrasound screening compared with clinical examination alone in the diagnosis and treatment of neonatal hip dysplasia in Austria. J Bone Joint Surg Br 2011;93 (08):1126-1130

12 Wynne-Davies R. Acetabular dysplasia and familial joint laxity: two etiological factors in congenital dislocation of the hip. A review of 589 patients and their families. JBone Joint Surg $\mathrm{Br}$ 1970;52(04):704-716

13 Mace J, Paton RW. Neonatal clinical screening of the hip in the diagnosis of developmental dysplasia of the hip: a 15-year prospective longitudinal observational study. Bone Joint J 2015; 97-B(02):265-269

14 Bache CE, Clegg J, Herron M. Risk factors for developmental dysplasia of the hip: ultrasonographic findings in the neonatal period. J Pediatr Orthop B 2002;11(03):212-218

15 Karol LA. Developmental dysplasia of the hip. In: Song KM. Orthopaedics Knowledge Update: Pediatrics 4. Rosemont, IL: American Academy of Orthopaedics Surgeons; 2011:159-68

16 Orak MM, Onay T, Gümüştaş SA, Gürsoy T, Muratlí HH. Is prematurity a risk factor for developmental dysplasia of the hip?: a prospective study Bone Joint J 2015;97-B(05):716-720 
17 Woodacre T, Ball T, Cox P. Epidemiology of developmental dysplasia of the hip within the UK: refining the risk factors. JChild Orthop 2016;10(06):633-642

18 Paton RW, Hinduja K, Thomas CD. The significance of at-risk factors in ultrasound surveillance of developmental dysplasia of the hip. A ten-year prospective study. J Bone Joint Surg Br 2005;87 (09):1264-1266

19 Williams D, Protopapa E, Stohr K, Hunter JB, Roposch A. The most relevant diagnostic criteria for developmental dysplasia of the hip: a study of British specialists. BMC Musculoskelet Disord 2016;17:38

20 Ortiz-Neira CL, Paolucci EO, Donnon T. A meta-analysis of common risk factors associated with the diagnosis of developmental dysplasia of the hip in newborns. Eur J Radiol 2012;81(03): e344-e351

21 Benson MKD, Macnicol MF. Developmental dysplasia of the hip. In: Benson M, Fixsen J, Macnicol M, Parsch K, eds. Children's orthopaedics and fractures. 3rd ed. London: Churchill-Livingstone; 2010:359-82

22 Donnelly KJ, Chan KW, Cosgrove AP. Delayed diagnosis of developmental dysplasia of the hip in Northern Ireland: can we do better? Bone Joint J 2015;97-B(11):1572-1576
23 Thomas SR. A review of long-term outcomes for late presenting developmental hip dysplasia. Bone Joint J 2015;97-B(06): 729-733

24 Ortolani M. Um segno poco noto c sua importanza per la diagnosi prococe de prelussazione congenita dell'anca. Pediatria (Napoli) 1937;45:129-136

25 Von Rosen S; von ROSEN. Diagnosis and treatment of congenital dislocation of the hip hoint in the new-born. J Bone Joint Surg Br 1962;44-B(02):284-291

26 Schwend RM, Schoenecker P, Richards BS, Flynn JM, Vitale M; Pediatric Orthopaedic Society of North America. Screening the newborn for developmental dysplasia of the hip: now what do we do? J Pediatr Orthop 2007;27(06):607-610

27 Arti H, Mehdinasab SA, Arti S. Comparing results of clinical versus ultrasonographic examination in developmental dysplasia of hip. J Res Med Sci 2013;18(12):1051-1055

28 Finne PH, Dalen I, Ikonomou N, Ulimoen G, Hansen TW. Diagnosis of congenital hip dysplasia in the newborn. Acta Orthop 2008;79 (03):313-320

29 Problemas Ortopédicos. In: Atenção à Saúde do Recém-Nascido: Guia para Profissionais de Saúde. Brasília: Ministério da Saúde; 2012 